\title{
Transparency and the pharmaceutical industry
}

\author{
RM Bracewell \\ Editor-in-Chief, Journal of the Royal College of Physicians of Edinburgh
}

The pharmaceutical industry (pharma for short) has a major impact on all our lives, as physicians, patients and citizens. However, pharma has come under increasing scrutiny and important concerns have been raised about how the industry conducts itself (especially in relation to clinical trials), and manages its relationships with physicians and healthcare systems.

\section{Drug development, profits and CME}

Many billions of pounds have been spent on developing new medications.' While some new drugs have revolutionised the treatment of diseases, others are 'me-too' medications offering no substantial benefit over existing agents. An important criticism is that the industry tends to focus on the diseases of the affluent, neglecting clinical issues that are more pressing in the developing world.

In many countries, the UK in particular, pharma is an important industry, generating significant profits - some argue on the back of excessively high prices. Pharma invests very heavily in research and development, contributing significantly to the 'Knowledge Economy'.

Many doctors have received CME directly or indirectly funded by the industry; pharma is a major sponsor of medical meetings and congresses. The College has developed transparent policies which enable it to receive support for our symposia and other CME events, but in a manner which would not be perceived to influence our independence or the prescribing practices of our members.

\section{Access to clinical trial data}

Carefully conducted and appropriately reported clinical trials are of fundamental importance in evidence-based medicine. This applies to both new and established treatments (see, for example, p29l-2 of this issue). It is estimated that about half of all clinical trials are not reported; ${ }^{2}$ this is a problem of which we have been aware since $1986,{ }^{3}$ if not earlier. Perhaps unsurprisingly, there is a bias to the publication of 'positive' trials. The selective reporting of trials to regulatory agencies and in the medical literature influences decisions about the licensing and use of pharmaceutical agents in clinical practice.

\section{Clinicaltrials.gov}

One attempt to address this problem is the requirement for trial protocols and results to be registered in publically available databases. Legislation was passed in the USA requiring, from 2008, that all new or ongoing clinical trials with at least one site in the USA be registered on clinicaltrials.gov within one year of completion. ${ }^{4}$ However laudable this aim, in practice most trials are not registered. ${ }^{5}$ This is an area in which audit may prove vital in raising - or rather enforcing - standards of behavior. ${ }^{6}$

\section{AllTrials.net}

The College is backing the international AllTrials campaign (http://www.alltrials.net/).

In brief, the campaign has several objectives:

I. Planned clinical trials should be prospectively registered, with a summary of the trial protocol, before the first participant is recruited.

2. Past trials not registered should be registered retrospectively.

3. A summary of results should be publicly available within one year of completion of the trial.

4. Summary results from all past trials of medicines currently in use should be made publicly available.

5. Trial sponsors or others who produce a full report for marketing authorisation or any other purpose should make this publicly available.

\section{Good pharma, bad pharma, better pharma?}

Our purpose in inviting a pharmaceutical physician to write an editorial (see p269-73) was not to 'redress the balance', given the weight of negative coverage of pharma in the press of late. Rather, we hoped to give you a chance to consider how the industry is addressing some of the concerns raised, and to encourage debate of these important issues. Naturally, our publication of this piece should not be taken as an endorsement by the JRCPE or the College of the views expressed.

\section{REFERENCES}

I Castellani J.Are clinical trial data shared sufficiently today? Yes. BMJ 2013; 347: fl88I. http://dx.doi.org//0.II36/bmj.fl88I

2 Song F, Parekh S, Hooper $L$ et al. Dissemination and publication of research findings: an updated review of related biases. Health Technol Assess 20 I0; 14: I-193. http://dx.doi.org//0.33 I0/hta I4080

3 Simes RJ. Publication bias: the case for an international registry of clinical trials. J Clin Oncol 1986; 4: I529-4I.

4 McCray AT, Ide NC. Design and implementation of a national clinical trials registry. J Am Med Inform Assoc 2000; 7: 313-23.

5 Prayle AP, Hurley MN, Smyth AR. Compliance with mandatory reporting of clinical trial results on ClinicalTrials.gov: cross sectional study. BMJ 2012; 344: d7373. http://dx.doi.org/I0.II36/ bmj.d7373

6 Goldacre B, Heneghan C. Improving, and auditing, access to clinical trial results. BMJ 20I4; 348: g2 I3. http://dx.doi.org/I0.II36/bmj.g2I3 